\title{
A Case of Rheumatoid Arthritis with Unilateral Knee Synovial Hypertrophy in Hemiplegia
}

\author{
Chan Woo Kim, M.D., Mi Jung Kim, M.D., Si Bog Park M.D., Seung Hoon Han, M.D. \\ Department of Rehabilitation Medicine, Hanyang University College of Medicine, Guri 471-701, Korea
}

\begin{abstract}
A 64-year-old woman suffering right hemiplegia came in with pain and swelling on her left knee, general weakness and poor oral intake for 2 months. On physical examination we were able to palpate a mass with irregular margin around the left suprapatellar area. From the results of the magnetic resonance imaging (MRI), synovial proliferative disease, infectious arthritis, or gouty arthritis was suspected. We performed a blood laboratory test to detect rheumatologic diseases, knee joint aspiration, and bone scan for differential diagnosis, and were able to diagnose rheumatoid arthritis (RA) from the results of blood laboratory, physical examination, and bone scan. Consequently, we started medications for controlling RA. Herein, we report a case of rheumatoid arthritis with unilateral knee synovial hypertrophy in hemiplegia. If a right hemiplegic patient has recurrent pain on the left knee and synovial hypertrophy, and fails to respond to treatment for osteoarthritis, early detection by evaluation for rheumatic disease is crucial to prevent severe sequelae influencing rehabilitation of hemiplegia.
\end{abstract}

Key Words Hemiplegia, Synovial hypertrophy, Rheumatoid arthritis

\section{INTRODUCTION}

Rheumatoid arthritis (RA) is a chronic, systemic inflammatory disorder of unknown cause that may affect many tissues and organs, but principally attacks synovial joints. Its symmetric and infiltrative nature causes pain, edema, and limitation of motion (LOM) of multiple joints. ${ }^{1}$ Some authors have reported that joints with LOM secondary to

Received October 3, 2010; Accepted March 30, 2011

Corresponding author: Seung Hoon Han

Department of Rehabilitation Medicine, Guri Hospital, Hanyang

University College of Medicine, 249-1, Gyomun-dong, Guri 471-701, Korea

Tel: +82-31-560-2380, Fax: +82-31-564-4654, E-mail: stephan72@naver com

(c) This is an open-access article distributed under the terms of the Creative Commons Attribution Non-Commercial License (http:// creativecommons.org/licenses/by-nc/3.0) which permits unrestricted noncommercial use, distribution, and reproduction in any medium, provided the original work is properly cited.

Copyright () 2012 by Korean Academy of Rehabilitation Medicine hemiplegia or peripheral neuropathy are less commonly affected by RA. ${ }^{2,3}$ We report a unique case of a hemiplegic patient who complained of severe pain and swelling on the unaffected knee joint and was ultimately diagnosed with RA.

\section{CASE REPORT}

A 64-year-old right hemiplegic woman suffering from pain and swelling in her left knee joint that had been aggravating for the last 2 months, impaired gait functions, general weakness, and poor oral intake, came in to the hospital. We took a physical examination of the patient and were able to detect edema, flare, oppressive pain, and palpated a mass with irregular margin around the left suprapatellar area. The left knee joint was found to have limited flexion LOM of $120^{\circ}$, but the sensory test on the knee joint was normal. The patient had a history of right hemiplegia due to cerebral hemorrhage that occurred 
20 years ago, but she had been able to perform activities of daily living (ADL) on her own, including gait. The patient had intermittent pain and swelling in the left knee, and was diagnosed with degenerative osteoarthritis at a private clinic 3 years ago. She was prescribed and treated with hyaluronic acid and steroid injection, but the symptoms repeatedly got better then worse in a cyclical way.

Simple radiograph on her left knee joint, which the patient took after admission to our hospital, showed exudative joint, tissue edema, narrowed patellofemoral and femorotibial joint (Fig. 1). MRI was taken to establish the image of the palpated mass during the physical exami- nation, which showed potential synovial proliferative disease, infectious arthritis, or rheumatoid arthritis (Fig. 2). Blood laboratory test, knee joint aspiration test, and bone scan were taken for differential diagnosis under consultation with the department of rheumatoid medicine. Joint aspiration test showed to be normal, which ruled out infectious arthritis, and the result of a bone scan showed multiple arthritis in both hands, left wrist, right elbow, both shoulders, and left knee joint (Fig. 3). Blood lab test showed $169 \mathrm{IU} / \mathrm{ml}(0-15 \mathrm{IU} / \mathrm{ml})$ of rheumatoid factor, anti-CCP 2,017 EU/ml (0-25 EU/ml), ESR 38 $\mathrm{mm} / \mathrm{hr}(0-20 \mathrm{~mm} / \mathrm{hr}), \mathrm{CRP} 1.8 \mathrm{mg} / \mathrm{dl}(0-0.3 \mathrm{mg} / \mathrm{dl})$, and
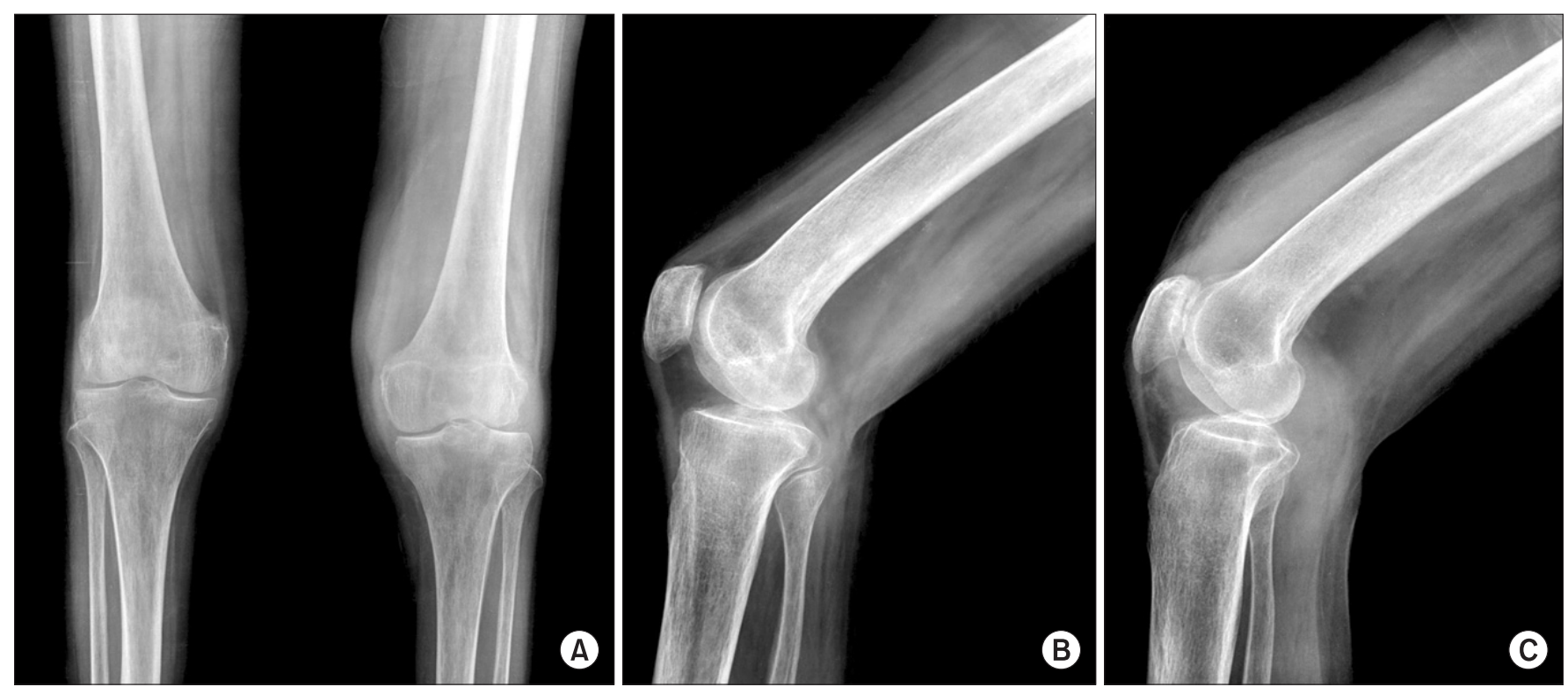

Fig. 1. Simple radiograph of left knee shows narrowing of the patellofemoral and femorotibial joint space. (A) AP view. (B) Right lateral view. (C) Left lateral view.
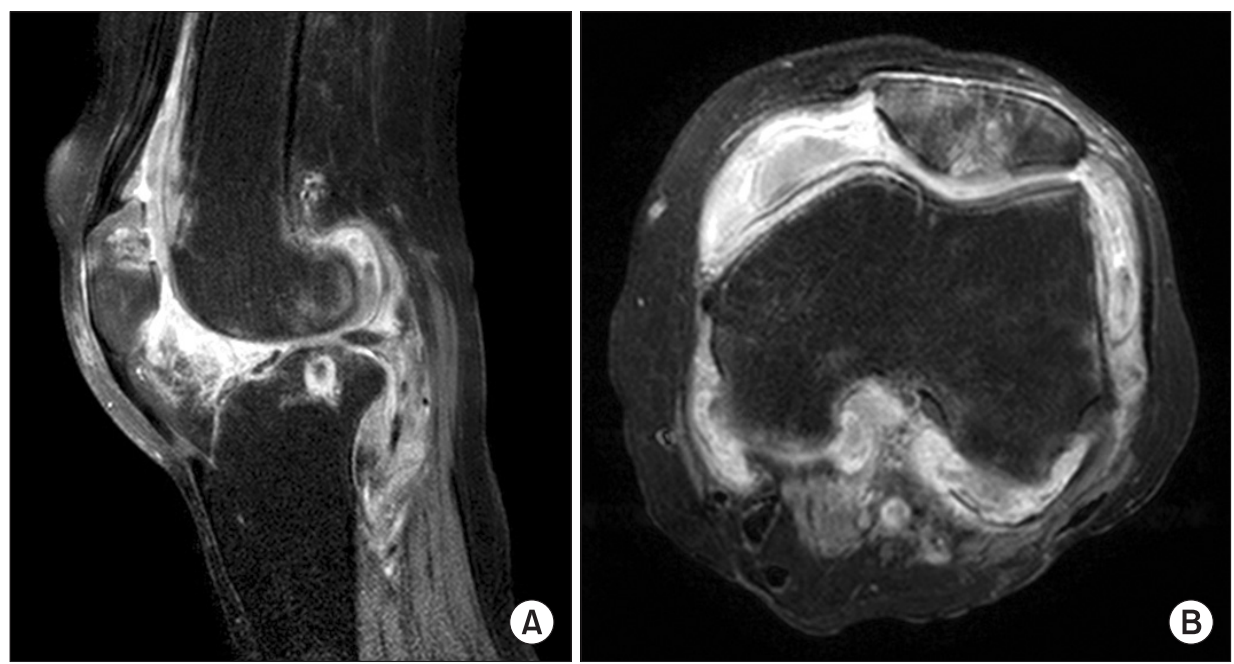

Fig. 2. Left knee MRI shows severe synovial hypertrophy and heterogenous contrast enhancement in hypertrophied synovium. (A) Sagittal view. (B) Transverse view. 


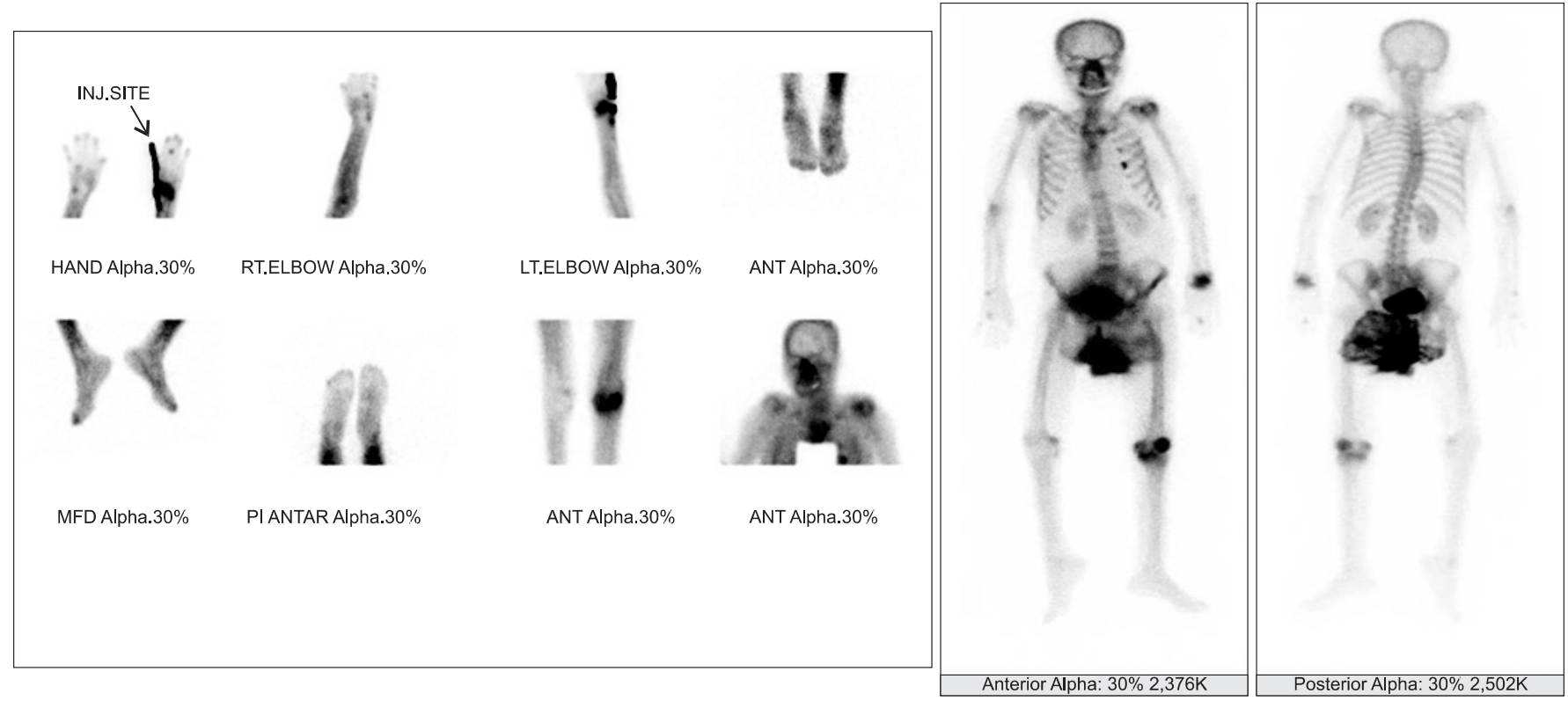

Fig. 3. Bone scan shows polyarthritis in both hands, left wrist, right elbow, both shoulders and left knee.

negative ANCA. The patient was diagnosed with RA for having more than three symptoms of the diagnostic criteria defined by American College of Rheumatology (ACR), which was established in 1987. The diagnostic criteria that the patient was diagnosed for RA includes morning stiffness, multiple arthritis observed in more than three parts of the body such as in the left wrist joint, left knee joint, and $4^{\text {th }}$ metacarpophalangeal joint of the right hand, plus arthritis in the hand, and positive rheumatoid factor found in the blood lab test. As the patient started treatment with hydroxychloroquine sulfate and cyclooxygenase-2 inhibitor, the symptoms of edema and pain in the left knee gradually decreased, and general weakness and poor oral intake also improved remarkably. The patient was recommended to have an immediate surgery for the synovial hypertrophy in the left knee, but she refused to do so. The surgery was then postponed until the patient's general condition was improved, following which she was discharged.

\section{DISCUSSION}

RA is a systemic autoimmune disease that usually features chronic symmetric multiple arthritis. The most important pathophysiology of RA is erosion in the cartilage and osseous tissue, which leads to chronic synovial hypertrophy in the joint and inflammatory reaction that destroys cartilage and its surrounding tissues. At the early stage, edema and pain in the joint develop, which triggers characteristic cartilage deformation and joint rigidity as the arthritis progresses. ${ }^{4}$

In addition, the incidence rate of stroke is recently increasing in young adults, and about $3.7-30 \%$ of the total stroke rate appears in juveniles and young adults of the age of $15-45 .{ }^{5}$ According to the previous study, stroke in young adults is known to have very diverse causes and risk factors compared to that of senescence, and young adults have a better ability to overcome the disabilities and exhibit higher neurologic and functional recovery rate after rehabilitation therapy compared to older patients. ${ }^{6}$ It is easy to find young stroke patients experiencing pain during active and aggressive rehabilitation therapy as seen in this case, and most clinicians often diagnose the patients with knee joint pain in the unaffected side as degenerative osteoarthritis due to excessive use of the joint, and treat them accordingly.

Thompson and Baywaters ${ }^{7}$ reported that RA is less developed in the paralytic side-joint compared to the sound side by describing four cases diagnosed with RA after stroke. Some studies reported that arthritis on the nonparalytic side was more affected than the paralytic side. Yaghmai et al., ${ }^{2}$ reported that 21 patients had less or no RA on the paralytic side, and Kamermann ${ }^{3}$ reported that there was no RA on the paralytic side after peripheral nerve injury.

The mechanism of the paralytic joint being protected 
from RA is not clear yet, but the common hypothesis is that lack of movement in the extremities has a prevention effect. It was hypothesized that because the unaffected side has to compensate for the paralytic side, increased use of the sound side accelerates and aggravates the risk and development of RA on the unaffected side. Norton and $\mathrm{Ziff}^{8}$ assumed that the immunity substances inside the joint such as fibrin or globulin complex promote inflammatory reaction by excessive use, aggravating the disease, and minimize inflammatory reactions in the paralytic side. Another hypothesis to explain the difference mentioned above is the internal pressure of the joint. Dixon and Grant ${ }^{9}$ stated that the incidence rate of arthritis would be different because the internal pressure in the paralytic joint is not as high as the unaffected side.

Synovium is the primary focus of RA, and synovitis is used as one of the indices for the activity level or the severity of the disease, along with exudative joint. Synovial hypertrophy such as exudative joint or papillary proliferation in the synovial joint can be observed. The characteristic synovial hypertrophy in the unaffected knee joint was also found in this case, and this type of synovial hypertrophy can be found in various infectious diseases and primary synovial diseases such as RA, degenerative arthritis, and pigmented villonodular synovitis (PVNS). ${ }^{10}$

Among these, PVNS is a proliferating disease, which originates from synovial joints, synovial cysts, and synovial sheath. It occurs in all age groups, and the most frequently affected joint is the knee. The joint fluid aspiration has bloody or dark brown color, low viscosity, and non-coagulating synovial fluid. The radiographic image differential is not clear, but the affected joint shows a swelling articular capsule, degenerative arthritic changes, and osteoclasia around the joint. MRI shows multiple lesions of heterogenic signal intensity due to lipid (fat, adipose cell) and hemosiderin along with synovial hypertrophy, and it can be distinguished from other diseases that have synovial hypertrophy based on this. In this case study, PVNS was also distinguished by MRI, the symptoms were treated, and the patient's condition improved through accurate diagnosis and treatment of RA.

When a hemiparetic patient has pain or edema in the unaffected knee joint, it is often diagnosed as degenerative osteoarthritis and treated accordingly. However, when the patient does not respond to continuous treatment for degenerative osteoarthritis and develops excessive synovial hypertrophy in the joint as seen in this case, the patient needs to be suspected of RA, and be appropriately evaluated. This process can detect and treat the disease earlier, and is the significant finding of this case report. It can also help to prevent severe sequelae influencing the rehabilitation of hemiplegic patients.

\section{REFERENCES}

1. Gabriel SE, Crowson CS, O'Fallon WM. The epidemiology of rheumatoid arthritis in Rochester, Minnesota, 1955-1985. Arthritis Rheum 1999; 42: 415-420

2. Yaghmai I, Roohomamini SM, Faunce HF. Unilateral rheumatoid arthritis: protective effect of neurologic deficits. Am J Roentgenol 1977; 128: 299-301

3. Kamermann JS. Protective effect of traumatic lesions on rheumatoid arthritis. Ann Rheum Dis 1966; 25: 361-363

4. Kim HY. Diagnosis and differential diagnosis of rheumatoid arthritis. J Korean Med Assoc 1986; 29: 15-20

5. Adunsky A, Hershkowitz M, Rabbi R, Asher-Sivron L, Ohry A. Functional recovery in young stroke patients. Arch Phys Med Rehabil 1992; 73: 859-862

6. Hindfelt B, Nilsson O. Long-term prognosis of ischemic stroke in young adults. Acta Neurol Scand 1992; 86: $440-445$

7. Thompson M, Baywaters EG. Unilateral rheumatoid arthritis following hemiplegia. Ann Rheum Dis 1962; 21: $370-377$

8. Norton WL, Ziff M. Electron microscopic observations on the rheumatoid synovial membrane. Arthritis Rheum 1966; 9: 589-610

9. Dixon AS, Grant C . Acute synovial rupture in rheumatoid arthritis. Clinical and experimental observations. Lancet 1964; 1: 742-745

10. Frassica FJ, Combs JJ Jr, Sim FH. Synovial proliferative disorders: differential diagnosis. Arhroscopy 1985; 1: 183-189 